\title{
Temps consacré par les adultes à l'évaluation PIAAC et importance de cette donnée
}

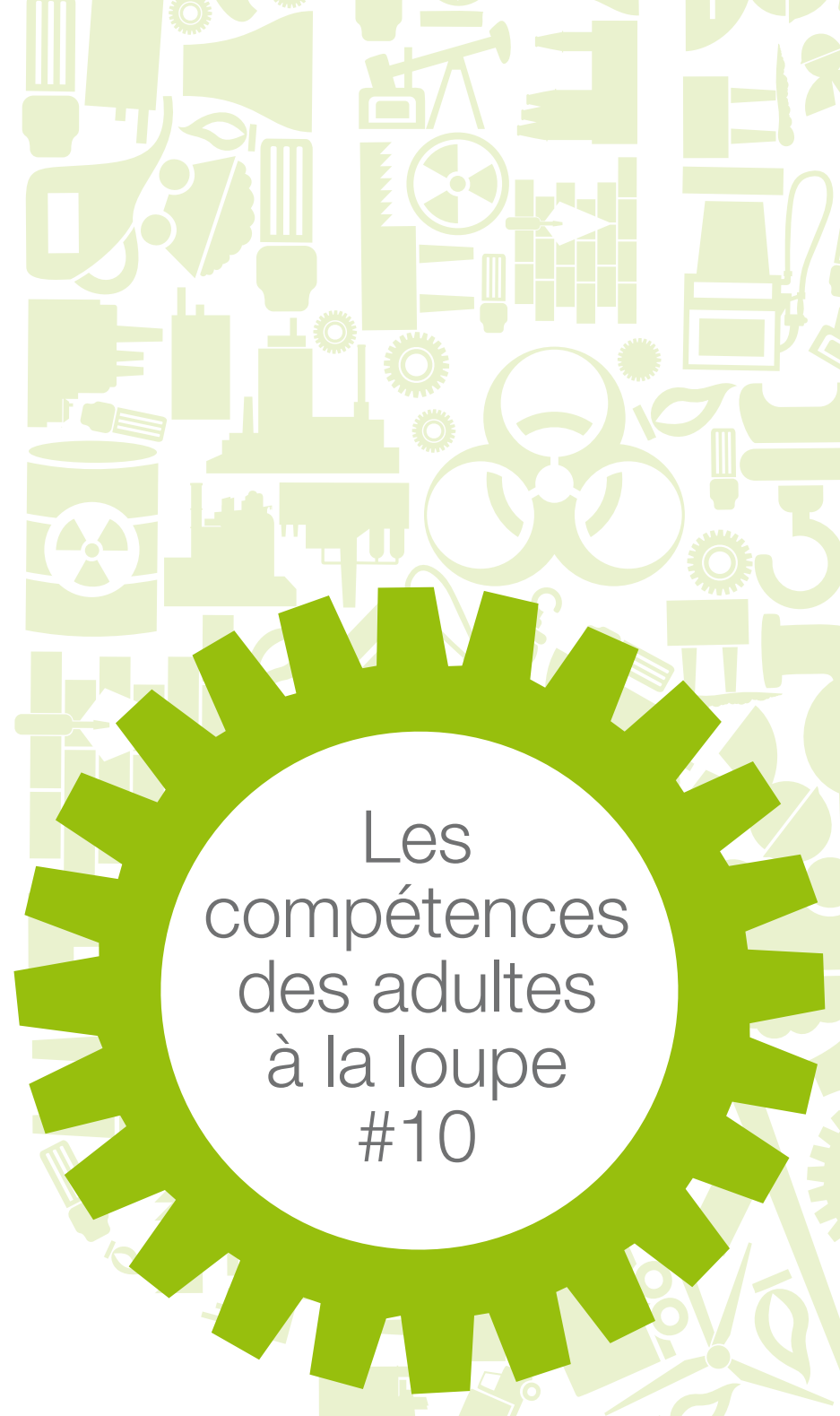




\section{LES COMPÉTENCES DES ADULTES}

- L'administration sur ordinateur (plutôt que sur papier) des évaluations telles que le PIAAC permet de recueillir des données sur l'interaction des répondants avec la plateforme d'évaluation, et notamment sur le temps qu'ils consacrent aux divers items et tâches.

- Ces informations supplémentaires, consignées dans des fichiers journaux, peuvent constituer un précieux complément aux traditionnelles données sur la performance des répondants. Elles peuvent être utilisées pour décrire les stratégies cognitives adoptées par les répondants et aider ces derniers à améliorer leurs résultats. Elles peuvent également servir à l'élaboration d'indicateurs d'engagement (pour mesurer les efforts déployés par les répondants) qui sont particulièrement importants pour l'interprétation de la performance dans les évaluations dont les enjeux sont peu élevés.

- Le temps consacré par les répondants à l'évaluation varie grandement d'un pays à l'autre. Il a tendance à s'accroître avec l'âge et le niveau de formation des répondants. Des différences similaires se dégagent quant à la fréquence du désengagement. Celui-ci est plus fréquent dans la deuxième moitié de l'évaluation.

L'administration informatisée d'évaluations à grande échelle, rendue possible grâce aux avancées technologiques et à la maîtrise croissante des ordinateurs et autres appareils numériques, renforce l'efficacité de l'administration des instruments et réduit le risque d'erreur humaine. Plus important encore, cela permet de collecter un ensemble plus complet de données sur les candidats à l'évaluation : les fichiers journaux consignent non seulement la réponse définitive à un item d'évaluation mais également toutes les interactions entre le répondant et la plateforme d'évaluation. Dans la mesure où tous les événements sont horodatés, il est possible de calculer le temps écoulé entre chacun d'entre eux.

L'interprétation de ces données se trouve compliquée par le fait que de nombreux processus cognitifs et non cognitifs adoptés par les répondants ne nécessitent pas d'interaction avec la plateforme d'évaluation. Cela est dû en partie au fait que la majorité des items d'évaluation n'a pas été conçue en vue de permettre l'enregistrement d'actions ou de processus spécifiques via la plateforme d'évaluation. Les prochaines évaluations tireront parti des possibilités offertes par les interfaces d'évaluation numériques et fourniront davantage de données à caractère informatif.

Toutefois, des indicateurs relativement simples tels que le temps consacré par les répondants aux items d'évaluation peuvent dès à présent conduire à des analyses utiles et fournir de précieuses informations venant compléter les données sur la performance globale à l'évaluation, tant à l'échelon national qu'individuel.

\section{Le temps que les répondants consacrent à l'évaluation varie grandement}

En Allemagne, en Autriche, en Finlande et en Norvège, les répondants ont consacré le plus de temps à la réalisation des épreuves de numératie et de littératie (avec une moyenne d'environ 50 minutes). Tandis qu'en
Angleterre et en Irlande du Nord (Royaume-Uni), en Espagne, en Irlande, en Italie et en République slovaque, les répondants y ont consacré en moyenne environ 40 minutes (graphique 1).

Graphique 1 / Temps total consacré aux tâches de numératie et littératie

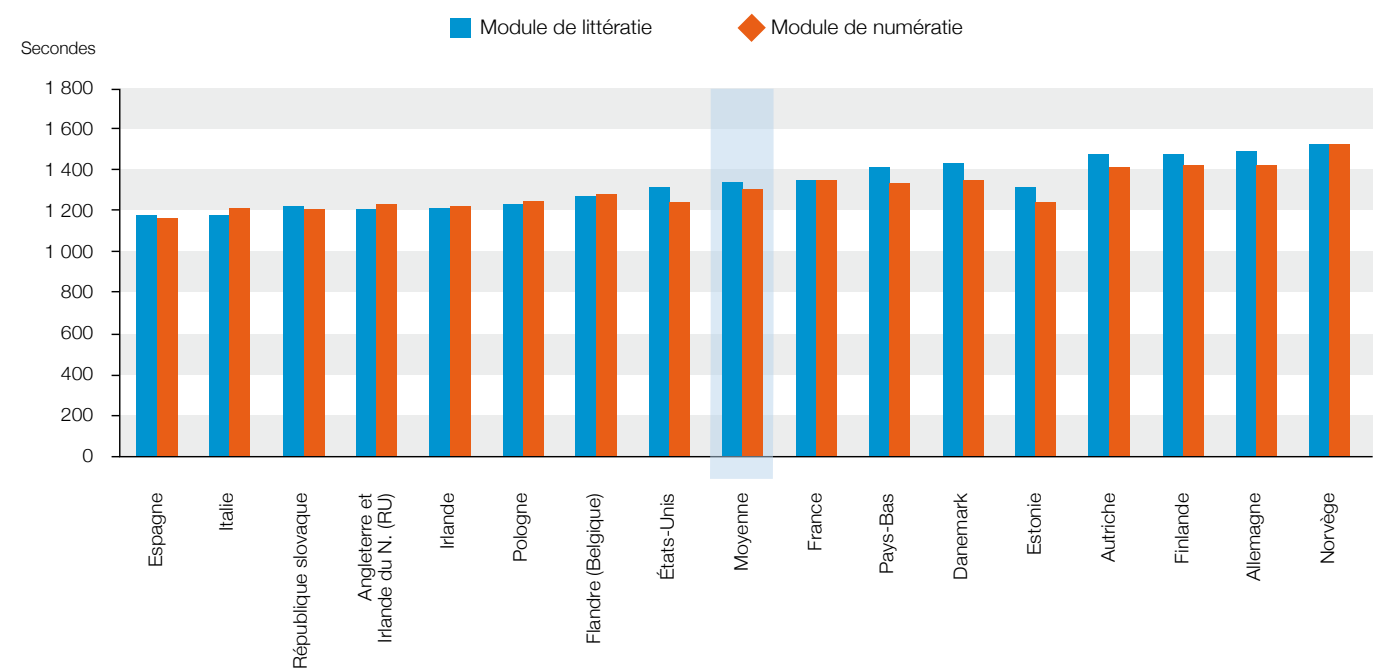

Remarque : l'échantillon ne comprend que les participants à l'évaluation informatisée qui ont dû effectuer les modules de numératie et de littératie.

Source : OCDE (2017), Programme pour l'évaluation internationale des compétences des adultes (PIAAC), Fichiers journaux. Archive des données de GESIS, Cologne. ZA6712 Version des fichiers de données 2.0.0, doi:10.4232/1.12955 
Une partie de ces écarts peut s'expliquer par le fait que différents items ont généralement été attribués à différents répondants : en effet, l'évaluation était adaptative en ce sens que les répondants les plus performants étaient plus susceptibles de devoir répondre à des items plus difficiles et inversement pour les répondants les moins performants. Une manière de prendre en compte la nature adaptative de l'évaluation consiste à calculer pour chaque individu et pour chaque item qui lui a été attribué un rang (exprimé en centile) dans la distribution du temps consacré aux tâches de chaque item, puis de calculer la moyenne de ces centiles dans les différents items. Étant donné que les écarts au niveau individuel se neutralisent à l'échelle nationale, cela a très peu d'incidence sur le classement des pays.
Plus intéressant encore, l'on observe des variations dans le temps consacré aux tâches en fonction des caractéristiques socio-démographiques des individus. Et notamment :

- les répondants avec le niveau de formation le plus élevé ont consacré davantage de temps à l'évaluation ;

- les répondants les plus âgés ont consacré davantage de temps à l'évaluation ;

- les répondants ayant déclaré mieux maîtriser les TIC ont consacré moins de temps à l'évaluation ;

- les femmes ont consacré à l'évaluation moins de temps que les hommes, mais les écarts entre les sexes à cet égard étaient généralement très faibles.

\section{De quelle manière les répondants allouent-ils leur temps aux différents items?}

Afin d'analyser les stratégies adoptées par les répondants quant à la manière dont ils répartissent leur temps au cours de l'évaluation, il est utile d'exploiter le fait que, dans les données disponibles, l'on observe le même répondant confronté à plusieurs items. Cela permet un maintien de toutes les caractéristiques individuelles ainsi qu'une comparaison du comportement d'un même individu entre les différents items.

La première question qui se pose est de savoir si les répondants adoptent une stratégie différente quant au temps qu'ils allouent aux items. Pour y répondre, il est possible de calculer pour chaque répondant son rang dans la distribution du temps consacré aux tâches pour chaque item. Chaque répondant sera ensuite caractérisé par la distribution de son rang sur l'ensemble des items auxquels il aura répondu. Aux répondants qui consacrent toujours à peu près autant de temps aux items (par rapport à d'autres répondants devant répondre aux mêmes items) sera associée une distribution du rang centile peu dispersée, tandis que pour les répondants qui consacrent un temps anormalement élevé sur certains items et anormalement faible sur d'autres la distribution sera plus dispersée. L'écart type moyen de ces distributions du rang centile pour chaque répondant avoisine les 20 centiles, ce qui indique un niveau relativement faible de régularité individuelle : il n'est pas rare que les répondants soient assez rapides sur certains items et plutôt lents sur d'autres. En revanche, comme le montre le graphique 2, les écarts entre les pays restent très faibles pour cet indicateur.

Graphique 2 / Distribution individuelle du temps consacré aux tâches

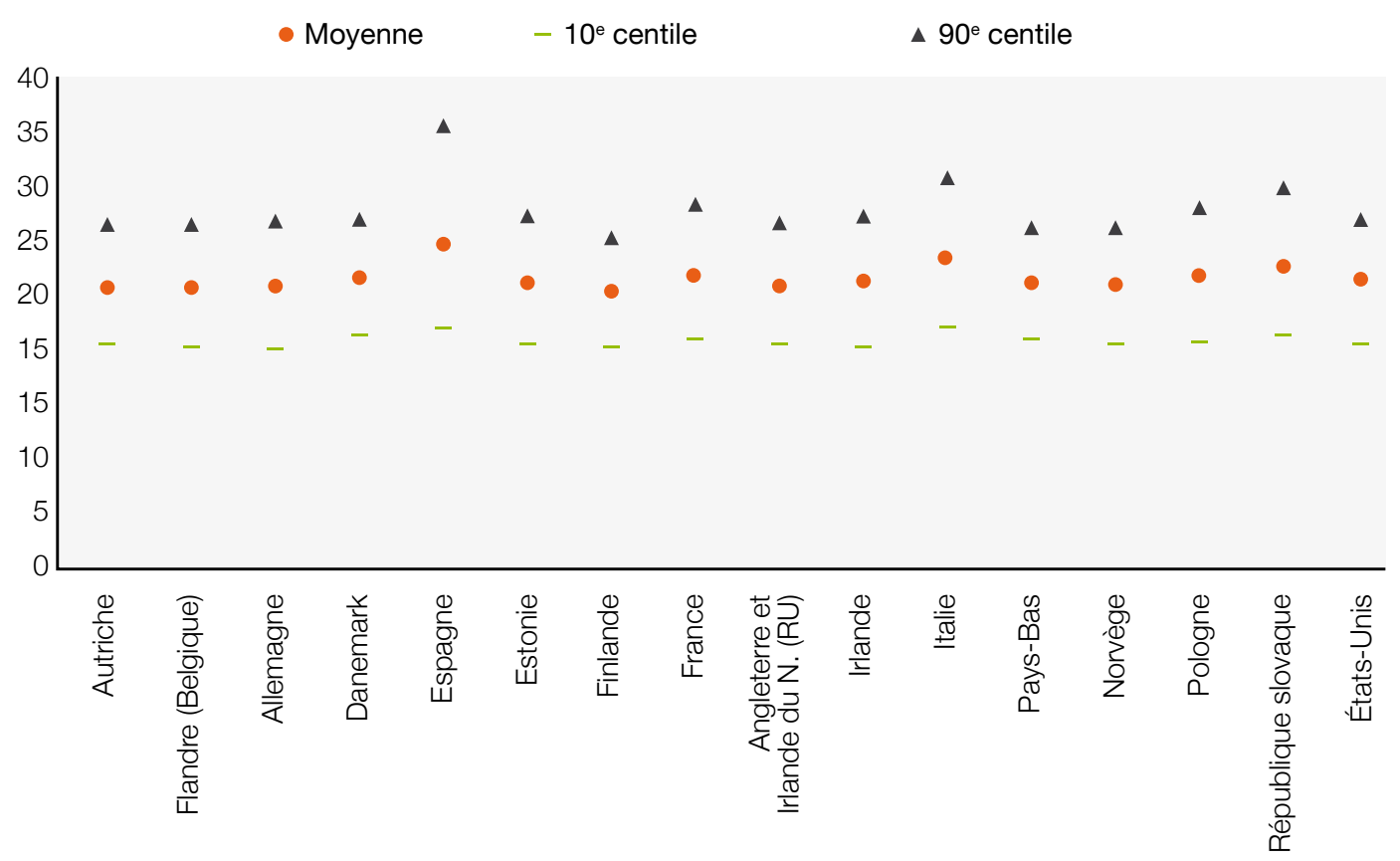

Remarque : le graphique montre des moments de la distribution au sein des pays des écarts types individuels dans le rang centile. L'échantillon ne comprend que les participants à l'évaluation informatisée qui ont dû effectuer les modules de numératie et de littératie.

Source : OCDE (2017), Programme pour l'évaluation internationale des compétences des adultes (PIAAC), Fichiers journaux, Archive des données de GESIS, Cologne. ZA6712 Version des fichiers de données 2.0.0, doi:10.4232/1.12955 


\section{LES COMPÉTENCES DES ADULTES}

À LA LOUPE

Il est également possible d'étudier la variation du temps consacré à un item en fonction de ses propres caractéristiques, comme son niveau de difficulté. Un individu qui attache de la valeur à son temps n'est pas censé consacrer beaucoup de temps à des questions trop difficiles et pour lesquelles il est donc peu probable qu'il réponde correctement. L'évaluation PIAAC permet de calculer au préalable une probabilité de réussite spécifique à un individu en associant aux informations sur les compétences des répondants le niveau de difficulté « objectif » et préétabli d'un item.

Le graphique 3 montre que plus les items deviennent difficiles par rapport au niveau de compétences des répondants, moins ces derniers y consacrent de temps (comparé à des répondants plus performants confrontés au même item). Réciproquement, le temps passé sur un item diminue également lorsque l'item est très facile pour le répondant, mais dans une moindre mesure. Cette asymétrie, où la diminution est plus conséquente pour les items très difficiles pour le répondant que pour les items très faciles s'explique simplement puisqu'un répondant confronté à un item très difficile sera tenté de l'abandonner rapidement sans en prendre entièrement connaissance, chose malgré tout nécessaire pour résoudre un item très facile.

Graphique 3 / Temps consacré aux tâches et probabilité relative de réussite

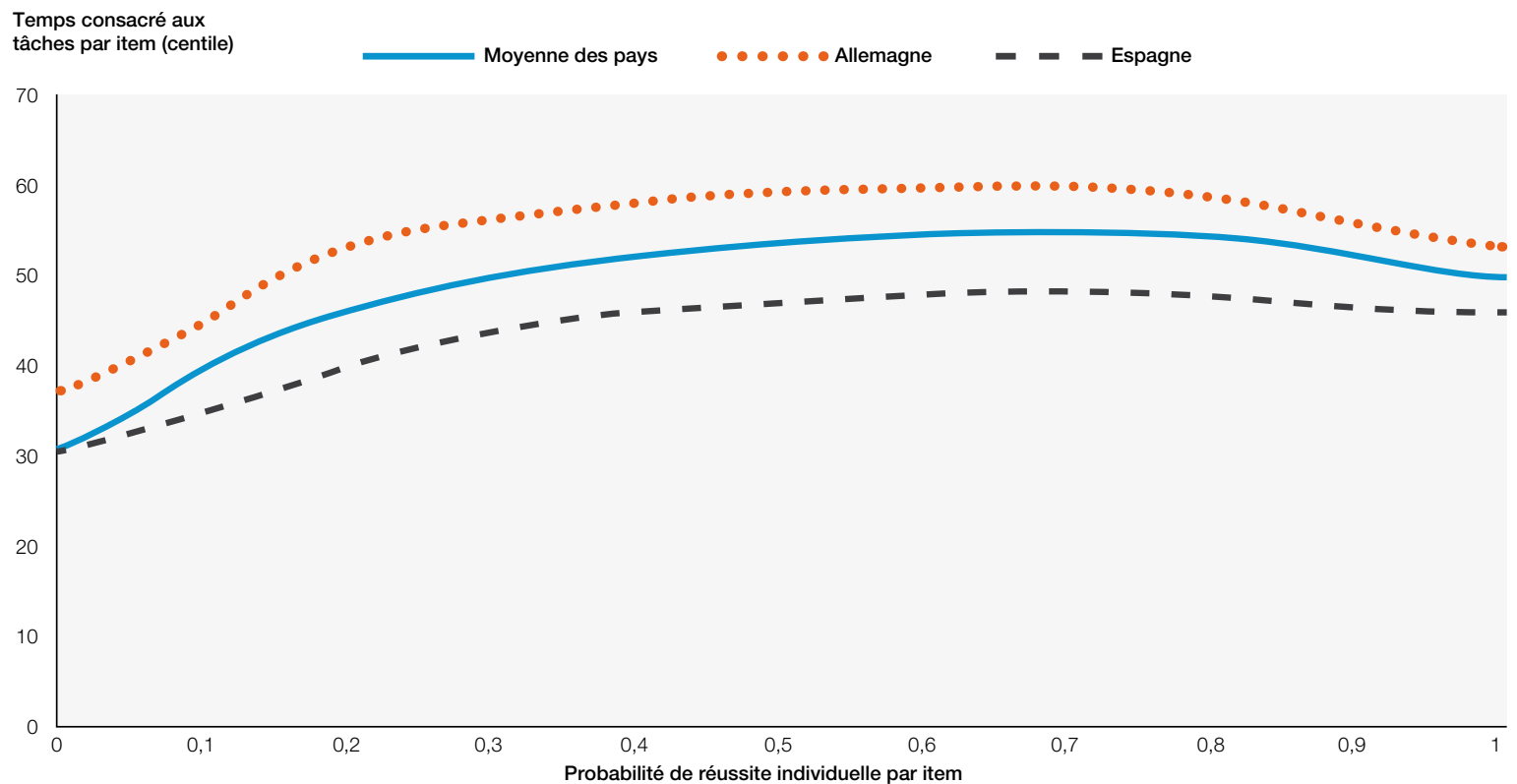

Remarque : l'échantillon ne comprend que les participants à l'évaluation informatisée qui ont dû effectuer les modules de numératie et de littératie.

Source : OCDE (2017), Programme pour l'évaluation internationale des compétences des adultes (PIAAC), Fichiers journaux, Archive des données de GESIS, Cologne. ZA6712 Version des fichiers de données 2.0.0, doi:10.4232/1.12955

La possibilité d'observer les réponses d'un même individu à plusieurs items permet également d'évaluer le lien de cause à effet entre le fait de consacrer davantage de temps à un item et la performance individuelle à l'évaluation, mesuré par la probabilité (ex post) de répondre correctement à l'item. En moyenne, le fait de consacrer une seconde supplémentaire sur un item augmente la probabilité de répondre correctement de 0.6 point de pourcentage.

\section{Dans quelle mesure les répondants prennent-ils l'évaluation au sérieux ?}

Le PIAAC est une évaluation dont les enjeux personnels pour le répondant sont peu élevés. Contrairement à des examens ou des concours, la performance aux épreuves du PIAAC n'a pas de conséquence pour les répondants qui, dès lors, n'ont aucune incitation externe à déployer de grands efforts. Si certains répondants ne prennent pas l'évaluation au sérieux et ne donnent pas le meilleur d'eux-mêmes, une source de variation préjudiciable (ou bruit) est introduite dans les estimations définitives du niveau de compétences d'un individu et d'une population. Si au sein des pays et entre ceux-ci divers sous-groupes de répondants font preuve de différents niveaux de désengagement, cela peut compromettre la validité des comparaisons entre les groupes de répondants et entre les pays.

Le lien entre le désengagement et la performance est une question complexe. Premièrement, le désengagement dans le PIAAC ne peut être mesuré qu'à partir d'indicateurs qui ne rendent compte que partiellement du spectre de désengagement. Deuxièmement, les liens qui existent entre le désengagement et la performance sont complexes et ne peuvent être facilement isolés. Enfin, les 
scores sur l'échelle des compétences obtenus au PIAAC prennent déjà partiellement en compte le désengagement en écartant les items auxquels les répondants consacrent moins de cinq secondes sans donner de réponse.

Une façon de déterminer qu'un répondant a fait preuve de désengagement à l'égard d'un item est de calculer, pour chaque item, le temps minimum nécessaire à y consacrer afin, au moins, de tenter de le comprendre et d'y répondre (Goldhammer et al., 2016). Les répondants qui consacrent moins de temps que ce seuil défini sont qualifiés de "désengagés » à l'égard de cet item particulier. Le graphique 4 indique les pourcentages de la population ayant fait preuve de désengagement à l'égard d'au moins $10 \%$ et d'au moins $20 \%$ des items. Si le désengagement concerne les répondants dans l'ensemble des pays, son ampleur est très variable. En effet, il est bien moins fréquent dans les pays d'Europe du Nord tels que la Finlande, la Norvège et les Pays-Bas. Dans ces pays, environ $8 \%$ de l'échantillon font preuve de désengagement à l'égard d'au moins 4 items sur 40 . Ce chiffre avoisine les $35 \%$ en Italie. Les mêmes écarts entre les pays se dégagent lorsque l'on observe des cas de désengagement plus importants, concernant au minimum $20 \%$ des items. La proportion chute sous la barre des $5 \%$ en Finlande, en Norvège et aux Pays-Bas, mais reste supérieure à $15 \%$ en Italie.

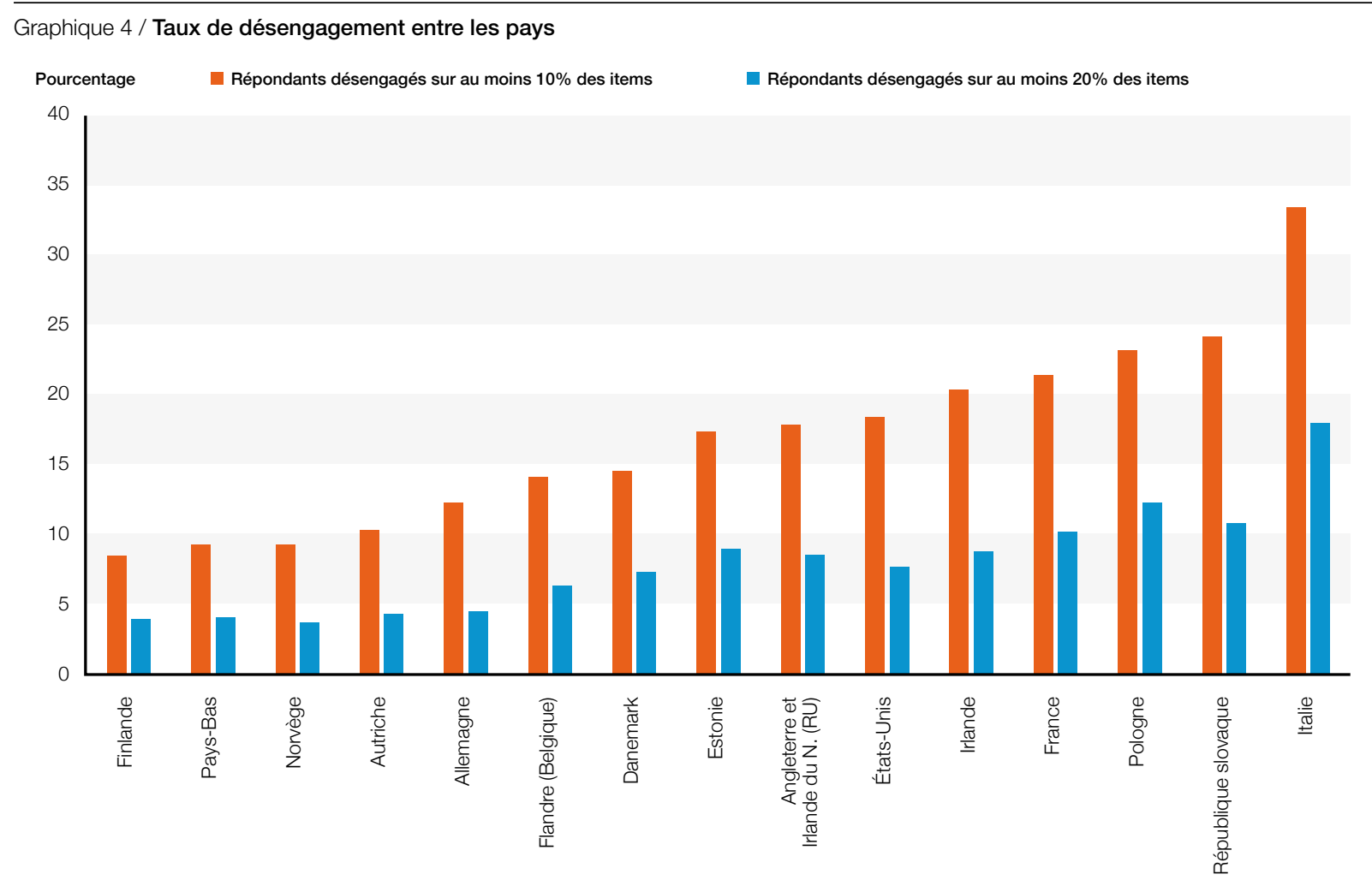

Source : OCDE (2017), Programme pour l'évaluation internationale des compétences des adultes (PIAAC), Fichiers journaux, Archive des données de GESIS, Cologne. ZA6712 Version des fichiers de données 2.0.0, doi:10.4232/1.12955

Les répondants sont plus susceptibles de faire preuve de désengagement par rapport aux items qui se trouvent dans la deuxième que dans la première partie de l'évaluation. Cette donnée est cohérente avec la tendance des répondants à consacrer moins de temps aux items du deuxième module. Cela pourrait en partie s'expliquer par le fait qu'au fur et à mesure de l'évaluation ils maîtrisent mieux l'interface utilisateur et sont donc plus rapides pour répondre aux items. Toutefois, la hausse des taux de désengagement apporte également la preuve que la motivation des répondants, ou leur capacité à fournir des efforts sur une longue période de temps, diminue en fonction de la longueur de l'évaluation.

Les indicateurs de désengagement sont très utiles à deux égards. Premièrement, ils contiennent des informations importantes sur le répondant et peuvent servir à évaluer un éventail de caractéristiques individuelles, comme le caractère consciencieux et la capacité à résister à la fatigue, des traits susceptibles d'influencer les résultats économiques et non économiques dans la vie. Deuxièmement, ces caractéristiques ne font pas partie des compétences généralement mesurées dans les évaluations cognitives. Ainsi, la présence de désengagement (ou tout type d'écart dans les efforts déployés par les répondants lors d'une évaluation) biaise les résultats des évaluations et peut poser des problèmes à l'heure de comparer les résultats à l'échelle internationale. À cet égard, les données relatives à l'ampleur du désengagement offrent un complément utile aux estimations réelles du niveau de compétence et peuvent permettre d'effectuer des comparaisons plus précises entre les pays. 


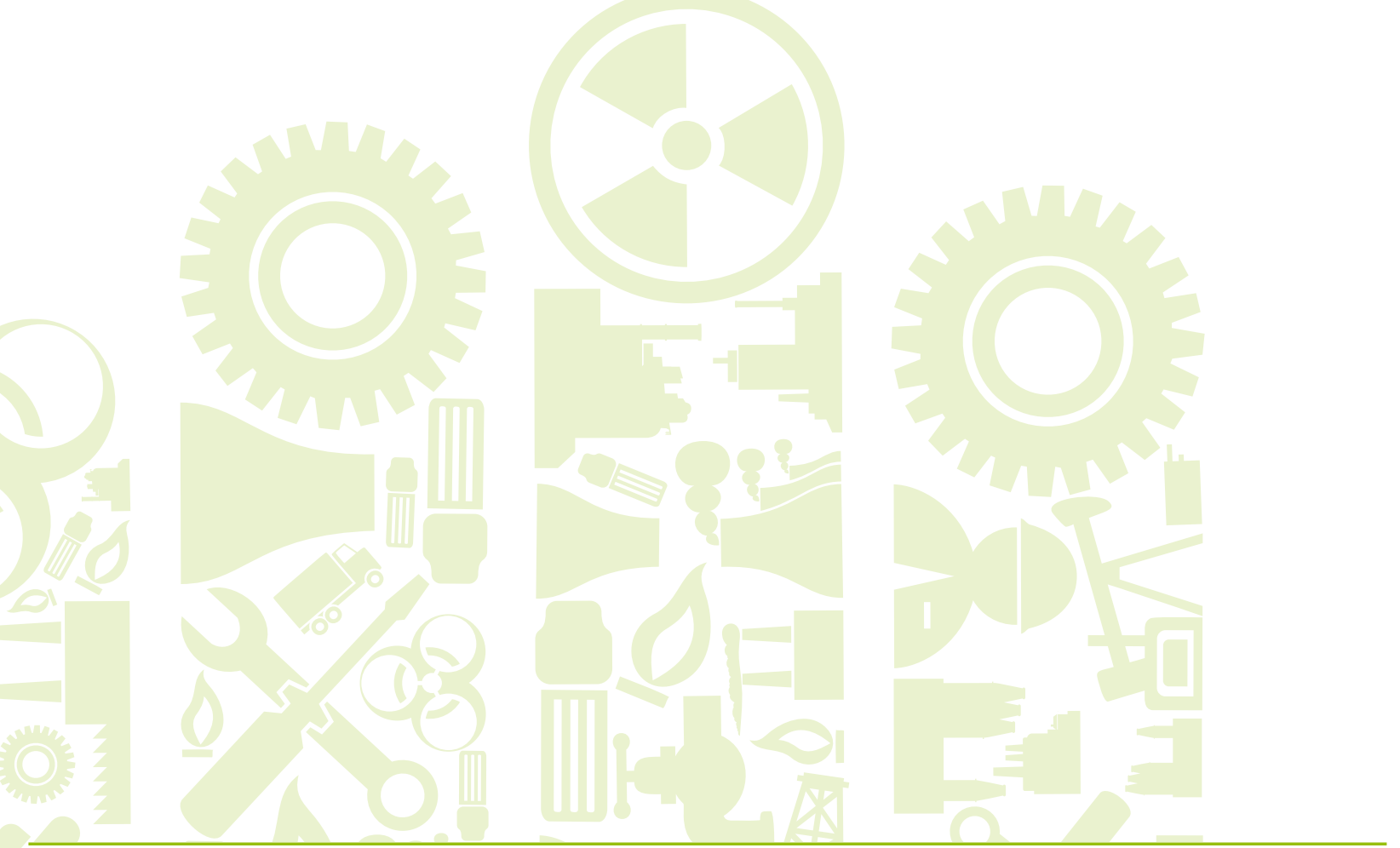

\section{Pour conclure}

Les fichiers journaux de l'évaluation informatisée peuvent contribuer à mieux comprendre les comportements des répondants et les stratégies cognitives qu'ils adoptent. L'analyse des données du PIAAC relatives au temps met en évidence d'importants écarts concernant le temps que consacrent les participants pour répondre aux items d'évaluation ainsi que des différences majeures au niveau des pays s'agissant du pourcentage de répondants qui consacrent le temps minimum nécessaire pour comprendre un item. Ces différences dans l'intensité des efforts déployés par les répondants offrent un complément utile à l'analyse des écarts de compétences à l'échelle internationale.

\section{$>$ CONTACT : \\ $>$ POUR EN \\ SAVOIR PLUS :}

\section{Marco Paccagnella (Marco.Paccagnella@oecd.org); (edu.piaac@oecd.org)}

OCDE (2019), Beyond Proficiency: Using Log Files to Understand Respondent Behaviour in the Survey of Adult Skills, Études de l'OCDE sur les compétences, Éditions OCDE, Paris, https://doi.org/10.1787/0b1414ed-en.

Goldhammer, F., et al. (2016), «Test-taking engagement in PIAAC», Documents de travail de l'OCDE sur l'éducation, $n^{\circ} 133$, Éditions OCDE, Paris, https://doi.org/10.1787/5jlzfl6fhxs2-en.

$>$ VISITER : www.oecd.org/skills/piaac

Indicateurs de l'éducation à la loupe - PISA à la loupe - L'enseignement à la loupe

L'Évaluation des compétences des adultes est un produit du Programme de l'OCDE pour l'évaluation internationale des compétences des adultes (PIAAC).

Ce document est publié sous la responsabilité du Secrétaire général de l'OCDE. Les opinions qui y sont exprimées et les arguments qui y sont employés ne reflètent pas nécessairement les vues officielles des pays membres de l'OCDE.

Ce document et toute carte qu'il peut comprendre sont sans préjudice du statut de tout territoire, de la souveraineté s'exerçant sur ce dernier, du tracé des frontières et limites internationales, et du nom de tout territoire, ville ou région.

Vous êtes autorisés à copier, télécharger ou imprimer du contenu OCDE pour votre utilisation personnelle. Vous pouvez inclure des extraits des publications, des bases de données et produits multimédia de l'OCDE dans vos documents, présentations, blogs, sites Internet et matériel d'enseignement, sous réserve de faire mention de la source OCDE et du copyright. Les demandes pour usage public ou commercial ou de traduction devront être adressées à rights@oecd.org.

Les données statistiques concernant Israël sont fournies par et sous la responsabilité des autorités israéliennes compétentes. L'utilisation de ces données par l'OCDE est sans préjudice du statut des hauteurs du Golan, de Jérusalem Est et des colonies de peuplement israéliennes en Cisjordanie aux termes du droit international. 